\title{
An Unusual Case of Antemortem and Postmortem Sex Offence with Homicide by a Juvenile Offender-A Case Report
}

\author{
Dr. Sayak Sovan Dutta ${ }^{1}$, Dr. Sandip Mukhopadhyay ${ }^{2}$, Dr. Sanchita Das Kundu ${ }^{3}$, \\ Dr. Arijit Dey ${ }^{4}$, Dr. Apurba Biswas ${ }^{5}$ Dr. Shelly Samanta ${ }^{6}$, \\ ${ }^{I}$ Demonstrator, Deptt of FSM, NRS Medical College, Kolkata \\ ${ }^{2}$ Demonstrator, Deptt of FSM, NRS Medical College, Kolkata \\ ${ }^{3}$ Assistant Professor, Deptt of FSM, NRS Medical College, Kolkata \\ ${ }^{4}{ }^{\text {rd }}$ Year PGT, Deptt of FSM, NRS Medical College, Kolkata \\ ${ }^{5}$ Assistant Professor, Deptt of FSM, Malda Medical College \\ ${ }^{6} 2^{\text {nd }}$ Year PGT, Deptt of FSM, NRS Medical College, Kolkata
}

\begin{abstract}
Sex offence to children is not uncommon now a days. Death following rape is also not unknown to us to prevent the victim to disclose the identity of the offender especially when the offender is known to the victim. Adult as well as juvenile sexual offenders against children exist in both inside and outside of our family system and posing a recognizable threat to vulnerable children. Sexual aberration and aggression reach its height when the offender after performing illegal sexual intercourse, kills the victim and even after death performs sexual intercourse with the dead body to achieve gratification, which is defined in term of 'Necrophilia'. Paedophilia is defined as recurrent, intense sexual fantasies, urges or behaviours involving sexual activity with a prepubescent child less than 13 years of age by a person who is 16 years or older and at least 5 years older than the child. Here in the following case a 7 year old girl was suffocated following rape and the assailant who was juvenile by age performed anal intercourse with the dead body to achieve gratification.
\end{abstract}

Keywords: sex offence, rape, sex offenders, necrophilia, juvenile, paedophilia, suffocation.

\section{Introduction}

History of the case as per information furnished by Police: On 28.1.15 the deceased who was 7 years of age went to play with her friends in the afternoon. In the evening she came back and after taking meal again went outside her house. She did not returned home. Her family members and local people searched her but could not found. On 29.1 .15 at $8.55 \mathrm{hrs}$ the deceased was found dead in the cultivation land 200 meter away from her home. The body was referred to NRSMedical College Hospital Morgue for PM examination from Dist. Hospital, Nadia on 29.01.15 and post mortem examination was done on the same day at $14.00 \mathrm{hrs}$.

\section{Findings On Post Mortem Examination:}

General description: One average built, moderately nourished body of female child. Rigor mortis was present all over the body (cool chamber).Eyes were closed, Corneas were hazy. Scalp hair 3" long and black in color. Complexion was medium. Wearing apparels were one black and red printed frock and one black full pant. One orange colour full sleeve jacket as present as belonging. Dried blood stain was detected surrounding genitalia and also present over wearing apparel at places. Mud and sand were present over buttock and posterior aspect of chest wall. There was evidence of bluish discolouration of nail beds. Sub-conjunctival petechial hemorrhage detected in both eyes.

External injury-(1) one abrasion 1"x0.5" present over left side of forehead, 2" left to midline, 0.3 " above left eyebrow. 2) one abrasion $0.4 " \mathrm{x} 0.3$ " present over tip of nose.3) one bruise 1 "x0.2" present over inner aspect of upper lip.4) one bruise 1.5"x0.3" present over inner aspect of lower lip.5) one lacerated wound 0.3 " $\mathrm{x} 0.2$ "x muscle present over inner aspect of lower lip, 0.4 " left to midline.6) one abrasion 2 "x 1 " present over anterior aspect of abdominal wall , 0.5 " left to midline, 3" above symphysis pubis.7)one linear scratch abrasion 2 " present over posterior aspect of left thigh, 4" below iliac crest, placed obliquely. 8) one linear scratch abrasion 2" present over posterior aspect of Left thigh, 6" below iliac crest, placed obliquely.9)one bruise 3" x2" present over medial aspect of left thigh 5" above knee joint. 10) one bruise 2"x2" present over medial aspect of right thigh 4" above knee joint. 11) abrasion measuring 2 "' 1 1" present over dorsal aspect of left hand over $2^{\text {nd }}$ and $3^{\text {rd }}$ metacarpophalangeal joint, 2.2 " proximal to tip of left index finger 12) one lacerated wound 1.5 " $x 0.5$ " $\mathrm{x}$ muscle present over anterior wall of anal canal without extravasation of blood in and around. 13) one lacerated wound 1 " 0.5 "x muscle present over posterior wall of vagina with extravasation of blood in and around.14) tear over 3 o'clock, 7 o'clock and 9 o'clock position over hymen with extravasation of blood in and around. 
On dissection-15) Numerous Petechial haemorrhagic spots were detected over visceral surface of both pleura and pericardium, interfaces of lobes of both lungs, visceral surface of heart. 16) Extravasation of blood diffused in left side of frontal region of scalp measuring 3 ' $\times 2$ "'

All internal organs were congested. Air passages contained blood stained frothy fluid. Lungs were congested and oedematous.

All the injuries were showing signs of vital reaction except injury no 12 which was post-mortem by nature .The margins of the lacerated wounds were irregular with extravasated blood in and around. The extravasated blood was bright red in colour. The abrasions were bright red, non-scabbed. No other injury could have been detected even after careful dissection and examination.

Materials preserved: 1 . viscera in saturated solution of common salt with control. 2. blood without any preservative. 3. blood soaked in bloating paper with control 4. nail cuttings and scrapping 5. plucked out tuft of scalp hair as control 6. combed out loose pubic hairs. 7. swab from vulva, perineum, inner side of thighs, anus, high and low vaginal swabs, cervix, urethra and also from mouth.

Cause of death was opined as Death was due to the effect of smothering- as noted above- ante mortem and homicidal in nature with evidence of Antemortem and postmortem sexual violence.

\section{Discussion}

Investigation revealed that the perpetrator was known to the victim since years and he was her neighbour. On the day of the incident the perpetrator who was 17 years old boy called the victim girl who was playing outside her house and lured her by offering chocolate. The girl did not think twice and followed him as she used to call her 'Bhaiya'. The boy then took her to the nearby cultivation land and raped her. During the course of rape when the girl started crying and shouting the assailant occluded her mouth and nose with his hand to supress her cry and smothered her and continued the act of intercourse even after the death of the child. After killing the child he returned home, took bath and returned after one hour to the cultivation land and performed anal intercourse with the dead body. On the next morning when the dead body of the deceased was discovered at the cultivation field by the local people and family members of the deceased he also joined them. But his unusual behaviour there created suspicion among the people who handed over him to the Police and later he confessed his crime to the Police.

Among legally identified cases of paraphilia, paedophilia is the commonest one. It is said that 10-20\% of all children have been molested by the age of $18{ }^{1}$ and $30-50 \%$ of all child sexual abuse is perpetrated by people under 21 years old (Vizard et al.1995) .

Pedophilia emerges before or during puberty, and is stable over time. It is said to be self-discovered, not chosen. For these reasons, pedophilia has been described as a disorder of sexual preference, phenomenologically similar to a heterosexual or homosexual sexual orientation. As per the definition of paedophilia whether the juvenile offender here could be stamped as a paedophile is not certain from a single act as criminal profiling of the assailant has not done yet to determine whether the urges or behaviour is recurrent which is most important for any paraphilia.

A person with paraphilia is supposed to have failed to complete normal and usual developmental process towards heterosexual adjustment. Work with adult and adolescent perpetrators of sexual abuse suggested that most of them have started the abusive behaviour even before puberty ${ }^{2}$. As per study of Graves in 1996, three categories of juvenile sexual offenders with distinct psychological profiles exist. These are:

1. The paedophilic offender 2. The sexual assault offender and 3. The mixed offence offender. Here in this case the assailant belongs to the no 3 by the nature of his crime.

There is no single theory which can explain the origin of sex offence in adolescent life. If we want to categorize the sexual behaviour of this juvenile offender it should be hyper sexualized, orgasm oriented and aggressive following Bremer classification.

There are seven types of adolescent sexual abusers: 1. group-influenced 2. disturbed impulsive 3. sexual compulsive 4. under socialized child exploiter 5. pseudo-socialized child exploiter 6. naïve experimenter and 7. miscellaneous.

It is highly required to perform criminal profiling of this juvenile offender for mapping the abuse i.e. his fantasies, strategies and behaviour for proper treatment and personal change.

Rosman and Resnick (1989) reviewed information from 34 cases of necrophilia describing the individuals' motivations for their behaviours among which $15 \%$ felt sexual attraction towards corpse and $12 \%$ was seeking self-esteem by expressing power over a homicide victim ${ }^{3}$. Though we don't know whether a single episode of intercourse with the deceased would stamp the juvenile assailant to be necrophilic, even if it is so he should be Homicidal necrophilic among ten-tire classification of necrophilia ${ }^{4}$. 
An Unusual Case of Antemortem And Postmortem Sex Offence with Homicide by A ..

Rosman and Resnick (1989) reviewed 122 cases of necrophilia. The sample was divided into genuine necrophiles, who had a persistent attraction to corpses, and pseudo-necrophiles, who acted out of opportunity, sadism, or transient interest ${ }^{5}$.History of the case here suggestive of the assailant might have transient interest as he also performed sexual intercourse with the victim girl when she was alive.

\section{Conclusion}

Media coverage of sexually aggressive adolescent or juvenile offenders has brought awareness among masses but not understanding the magnitude of the problem with the offenders which essentially highlights the need for professional assessment of the risk. Cognitive behavioural concepts can be used for reconstruction of treatment programmes for these juvenile sexual offenders. The 'STEP' programme which is cognitive behavioural based but uses a modified relapse prevention approach to the developmental needs of the young perpetrators can be used in management in these cases.

[1]. Subrahmanyam BV, Forensic Psychiatry, $1^{\text {st }}$ edition, p.135

\section{Reference}

[2]. Subrahmanyam BV, Forensic Psychiatry, $1^{\text {st }}$ edition, p. 132

[3]. Rosman, J. P.; Resnick, P. J. (1 June 1989). "Sexual attraction to corpses: A psychiatric review of necrophilia" (PDF/HTML). Bulletin of the American Academy of Psychiatry and the Law 17 (2): 153-163

[4]. Aggrawal, Anil (2009). "A new classification of necrophilia". Journal of Forensic and Legal Medicine 16 (6): 31620. doi:10.1016/j.jflm.2008.12.023

[5]. Rosman, J. P.; Resnick, P. J. (1 June 1989). "Sexual attraction to corpses: A psychiatric review of necrophilia" (PDF/HTML). Bulletin of the American Academy of Psychiatry and the Law 17 (2): $153-163$.

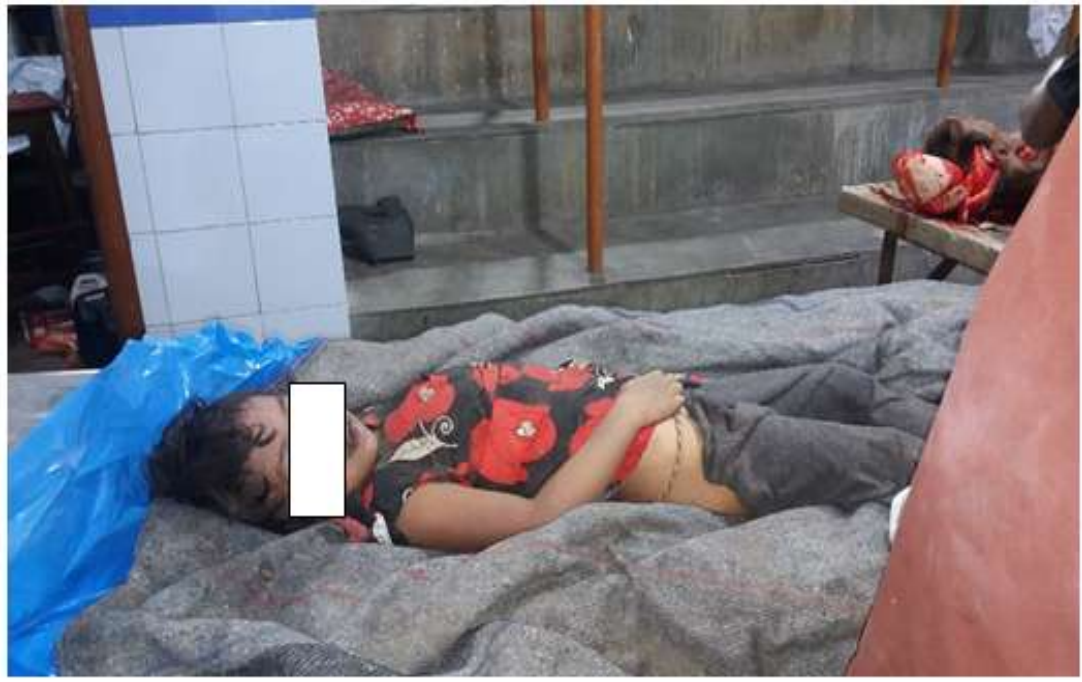

Photograph 1. External appearances of the deceased with wearing apparels

Photograph 2. Showing Bruise over Lower lip and tip of nose marked with arrow.

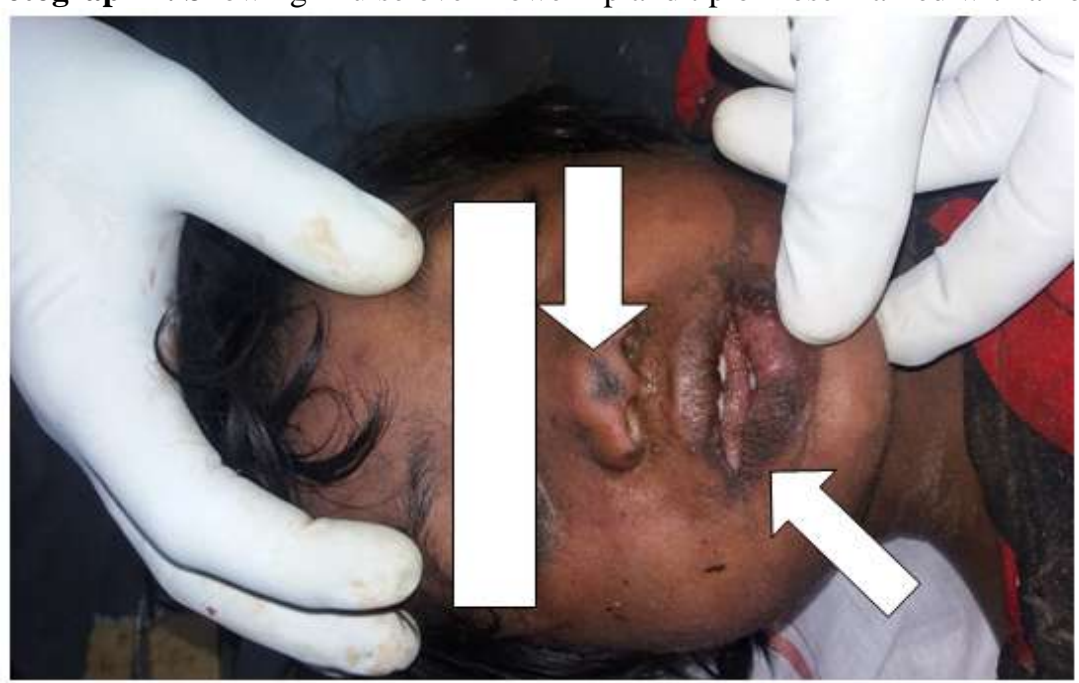




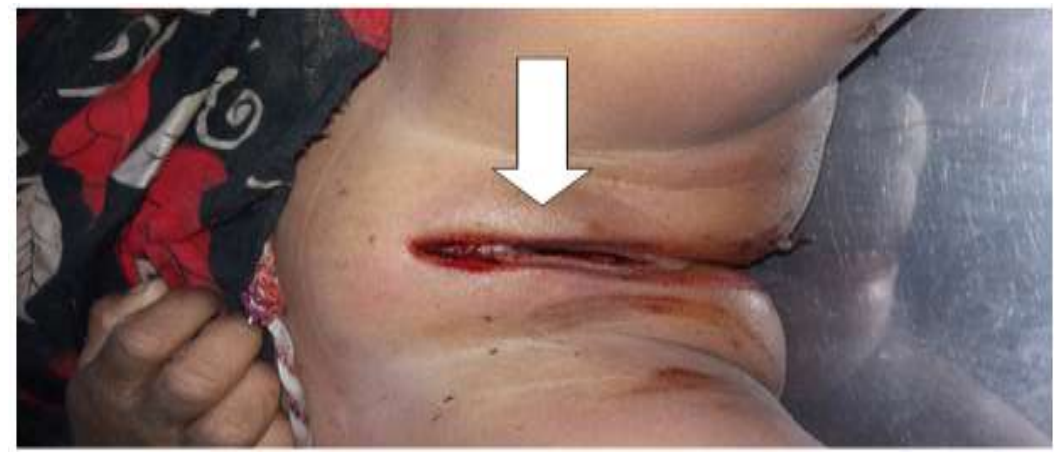

Photograph 3. Showing blood around external genitalia marked with arrow

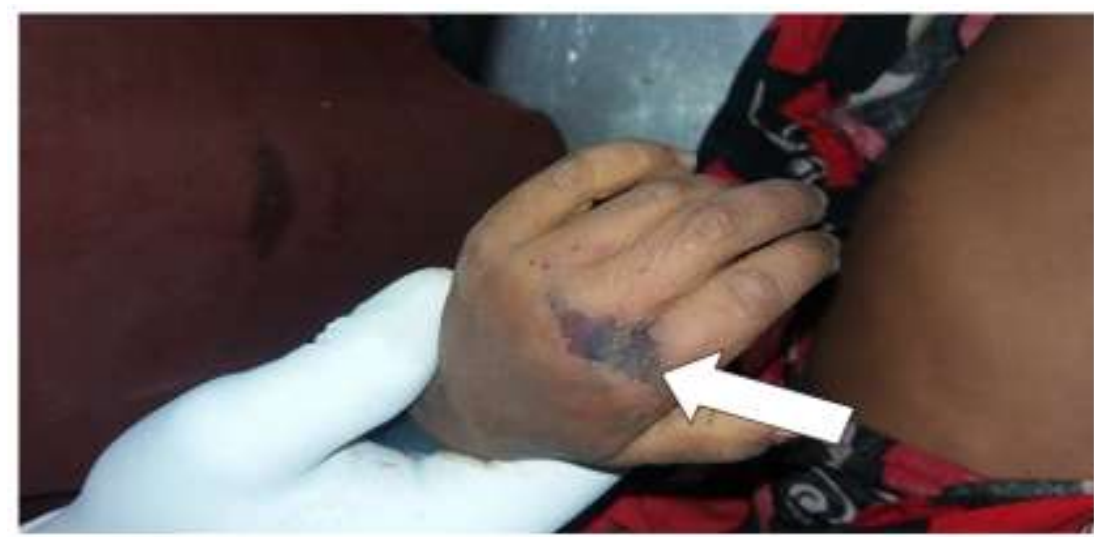

Photograph 4. Showing abrasion over dorsum of hand marked with arrow

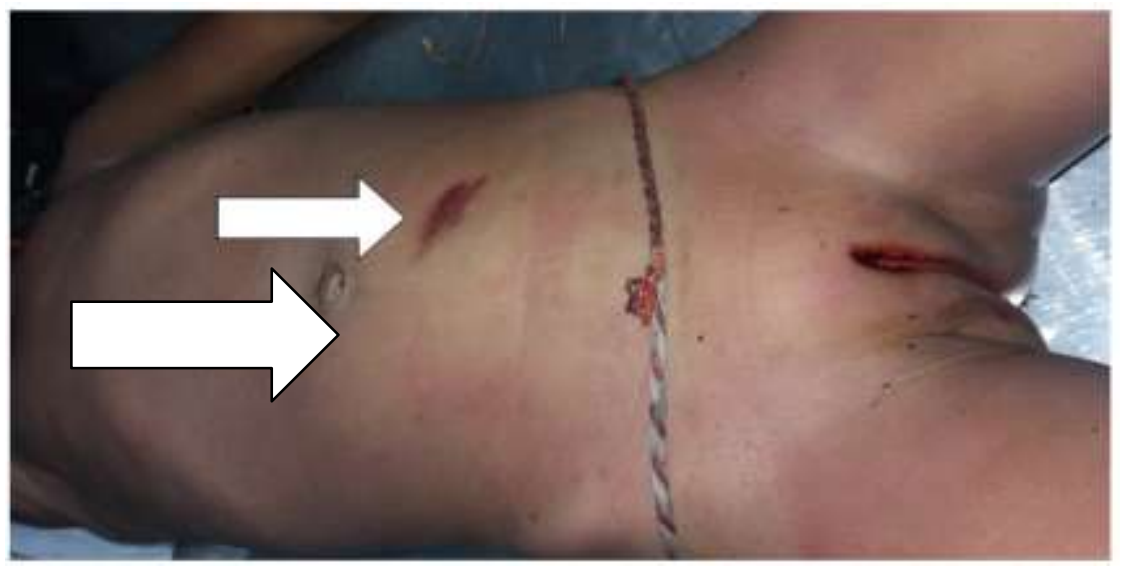

Photograph 5. Showing abrasion over anterior aspect of abdominal wall marked with arrow

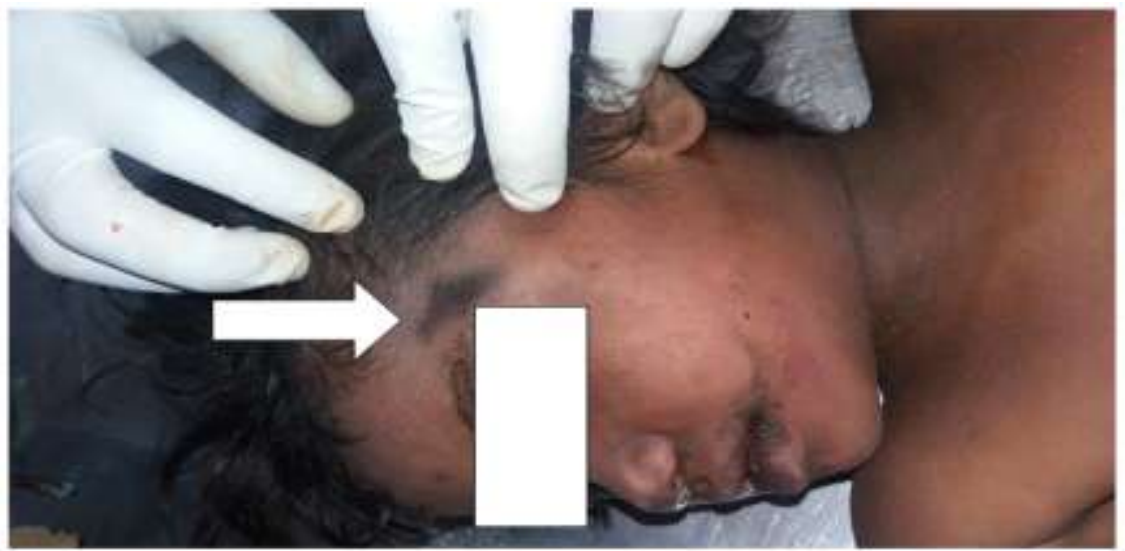

Photograph 6. Showing abrasion over left side of forehead at temple marked with arrow 


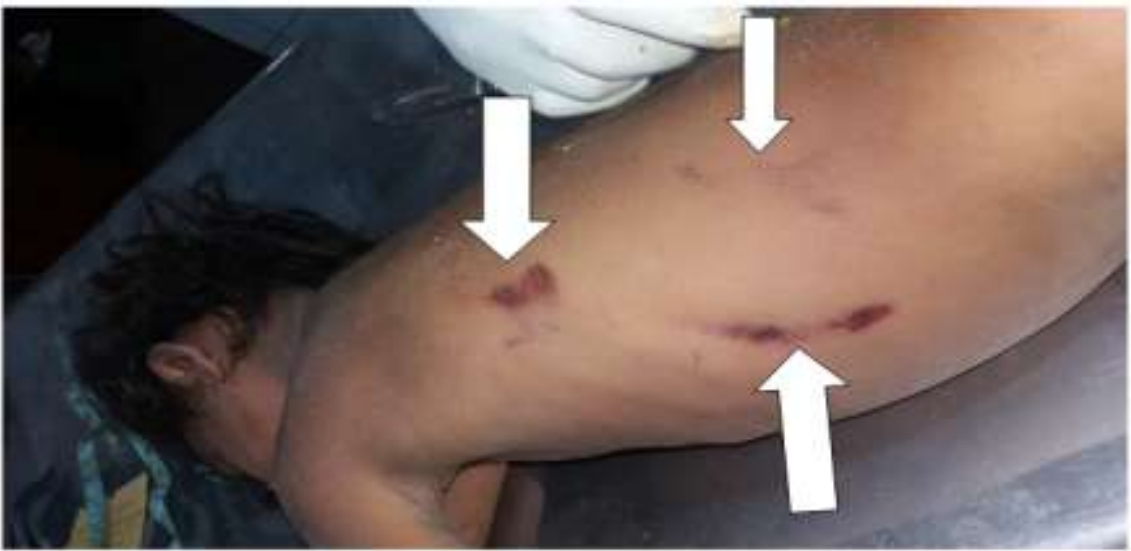

Photograph 7. Showing multiple abrasion over posterior and lateral aspect of chest and abdominal wall marked with arrow

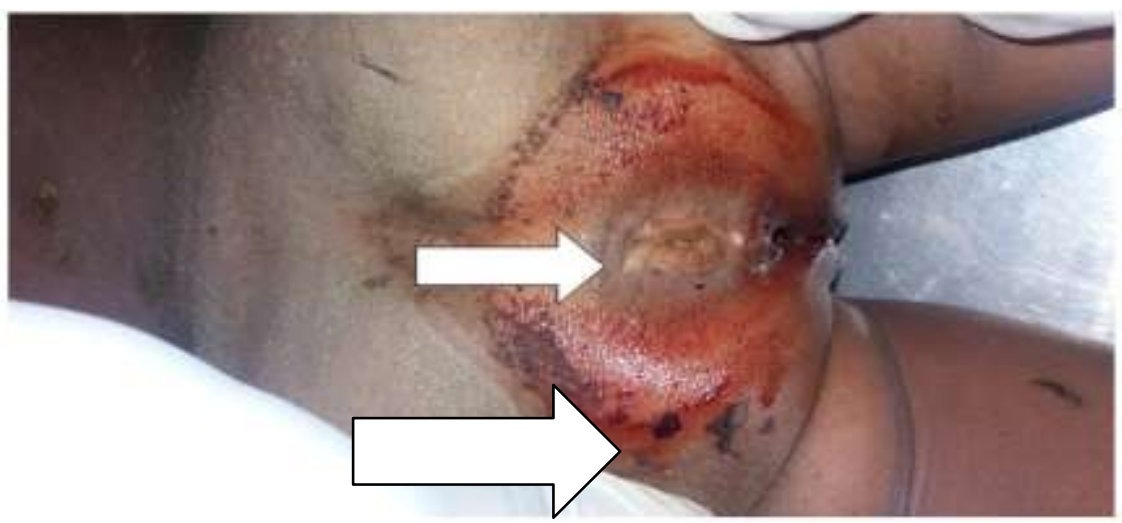

Photograph 8. Showing laceration of anal canal, Postmortem in nature, margins are not showing vital reaction marked with arrow (the local area stained with blood coming from vagina)

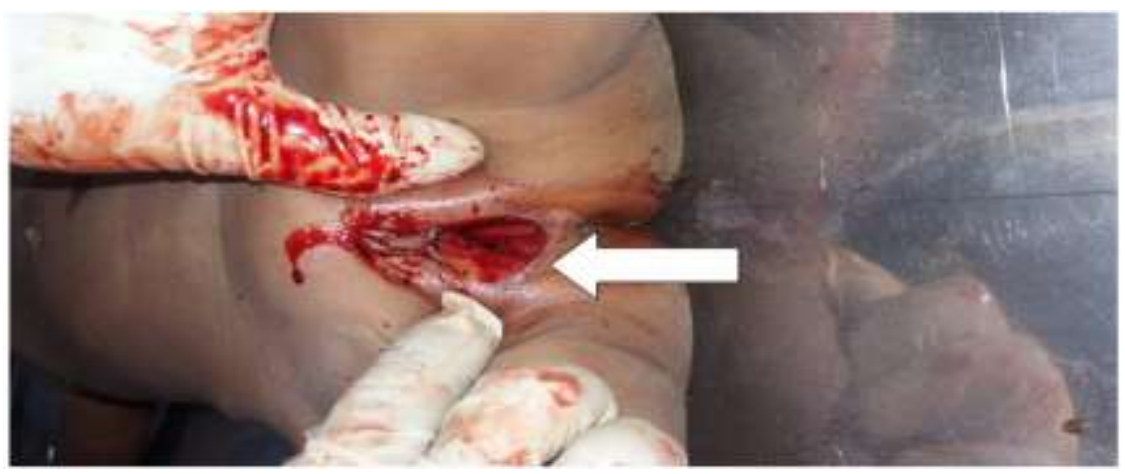

Photograph 9. Showing genital injury with extravasation of blood marked with arrow

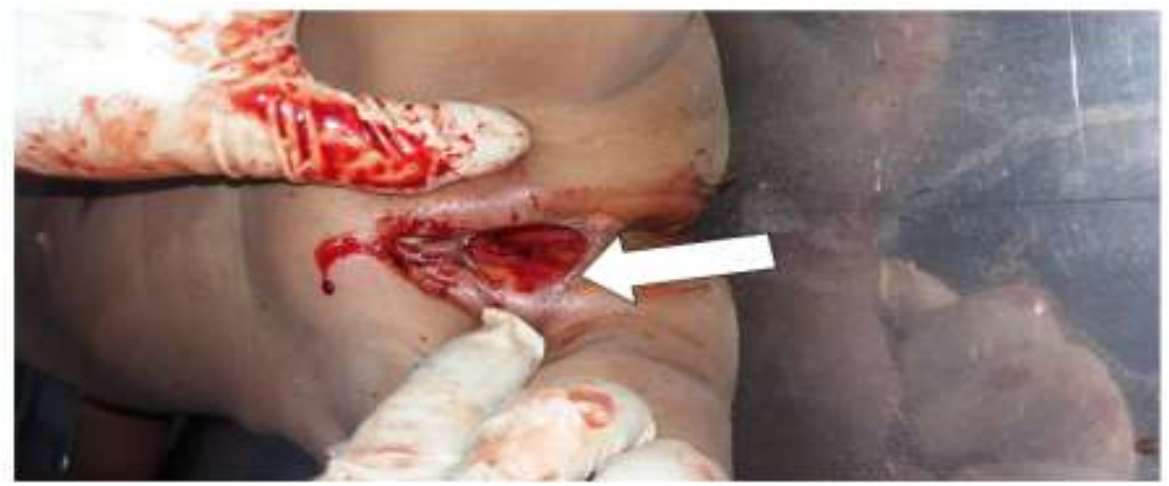

Photograph 10. Showing genital injury with multiple tear in hymen marked with arrow 


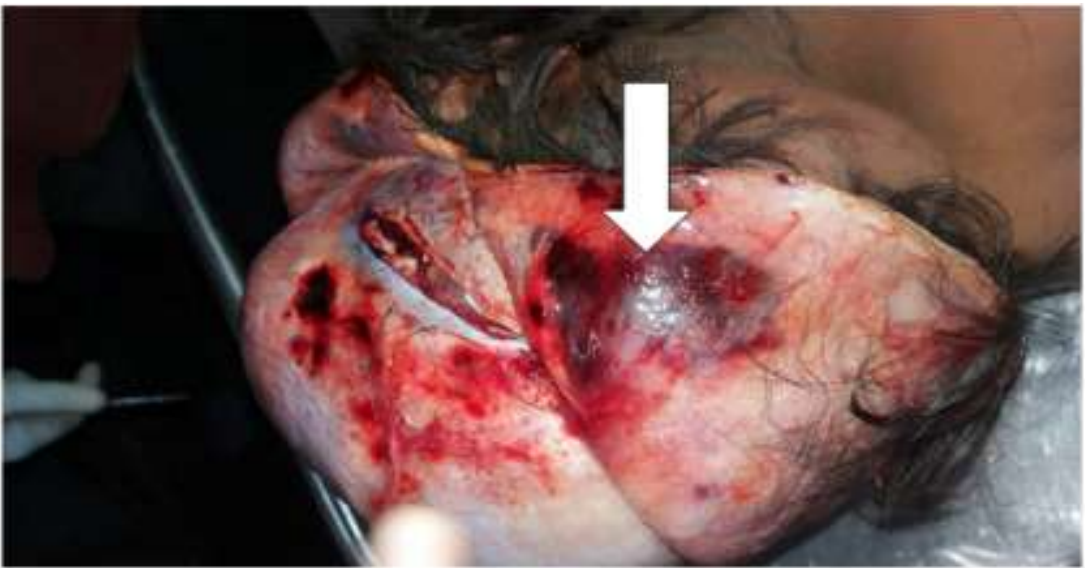

Photograph 11. Showing extravasation of blood into layers of scalp marked with arrow

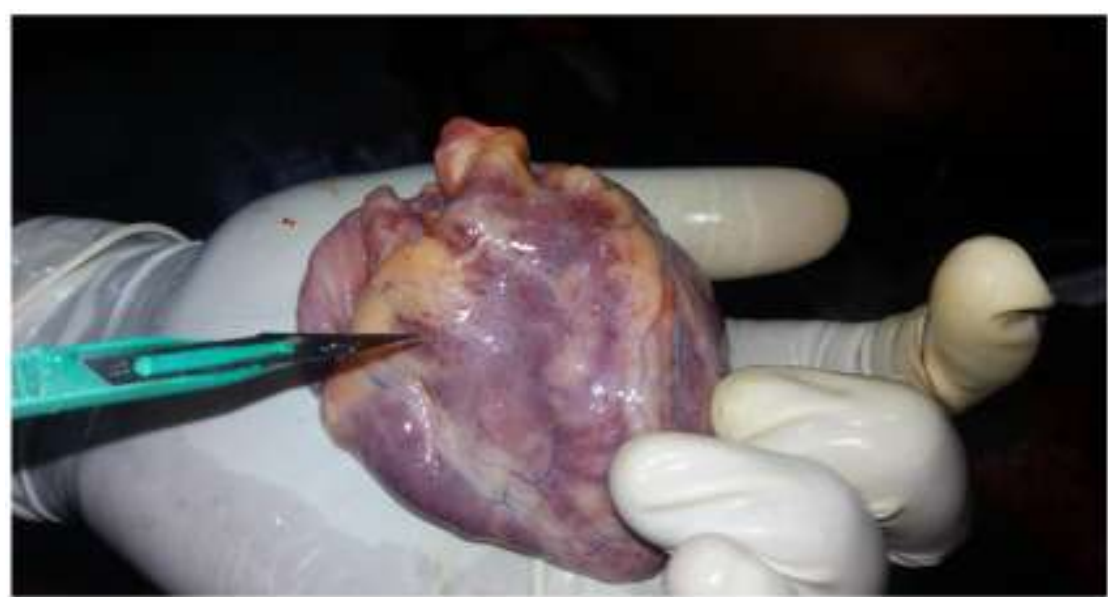

Photograph 12. Showing multiple petechial hemorrhagic spots present over visceral surface of heart pointed with tip of knife 